\title{
On Permutation Symmetries of Hopfield Model Neural Network
}

\author{
JIYANG DONG*, SHENCHU XU, ZHENXIANG CHEN and BOXI WU \\ Department of Physics, Xiamen University, 361005 Xiamen, P. R. China
}

(Received 29 July 2000)

\begin{abstract}
Discrete Hopfield neural network (DHNN) is studied by performing permutation operations on the synaptic weight matrix. The storable patterns set stored with Hebbian learning algorithm in a network without losing memories is studied, and a condition which makes sure all the patterns of the storable patterns set have a same basin size of attraction is proposed. Then, the permutation symmetries of the network are studied associating with the stored patterns set. A construction of the storable patterns set satisfying that condition is achieved by consideration of their invariance under a point group.
\end{abstract}

Keywords: Discrete hopfield neural network; Permutation symmetries; Associative memory; Storable patterns set

PACS numbers: 42.79.Ta, 84.35. $+\mathrm{i}, 11.30 . \mathrm{Na}$

\section{INTRODUCTION}

Symmetry plays an important role in unification and classification of physical systems [1], e.g., crystals by their point group symmetry or the atomic nucleus by the isospin symmetry. Permutation symmetry may also be important, a prominent example is the replica symmetry breaking in the spin glass theory, where a classification of the mean field theoretical solutions is achieved by consideration of their invariance under subgroups of the symmetric group [2].
Another object for which the study of symmetries proves to be very fruitful is the area of neural networks. Global properties of an individual network can be found from symmetry considerations of the invariance group of the specific pattern set stored by some learning rules $[3,4]$.

Here, the permutation symmetries on DHNN are associated with that on the stored patterns set. The organization of this paper is as follows. Section 2 gives a simple introduction on the discrete Hopfield neural network. In Section 3, the DHNN is studied by performing permutation operations on the

*Corresponding author. e-mail: jydong@jingxian.xmu.edu.cn 
synaptic weight matrix, and some interesting properties are found. The permutation symmetries of the network are studied associating with the stored patterns set. A condition that makes sure all patterns of the storable patterns set have a same basin size of attraction is proposed. In Section 4, a construction of the storable patterns set with same basin size of attraction is achieved by consideration of their invariance under a point group. We give some discussions and a conclusion in Section 5.

\section{DISCRETE HOPFIELD NEURAL NETWORK}

Discrete Hopfield neural network [5] (DHNN) was proposed mainly as an associative memory model. It is a dynamical system uniquely defined by $(W, \theta)$. Here $W$ is a symmetric zero-diagonal real weight matrix, where element $W_{i j}$, denotes the weight of the connection from neuron $j$ to neuron $i$ $(i, j=1,2, \ldots, N$ for $N$-neurons network), $\theta$ is a vector of dimension $N$, where component $\theta_{i}$ denotes the threshold value of neuron $i$. The state of neuron $i$ is denoted by $X_{i}(t)$ and it is either " 0 " or " 1 ". The state vector of the DHNN at time $t$ is denoted by $X(t)=\left[X_{1}(t), X_{2}(t), \ldots, X_{N}(t)\right]^{t}$. The state of neuron $i$ at time $t+1$ is computed by

$$
X_{i}(t+1)=\operatorname{step}\left(\sum_{j=1}^{N} W_{i j} X_{j}(t)-\theta_{i}(t)\right) \quad \forall i
$$

here,

$$
\operatorname{step}(x)= \begin{cases}1 & x \geq 0 \\ 0 & x<0 .\end{cases}
$$

Without losing of generality, we let $\theta_{i}=0$, $i=1,2, \ldots, N$ for the sake of simplicity in this paper.

A state $X=\left(X_{1}, X_{2}, \ldots, X_{N}\right)^{t}$ of the network is called stable if

$$
X_{i}=\operatorname{step}\left(\sum_{j=1}^{N} W_{i j} X_{j}-\theta_{i}\right) \quad(i=1,2, \ldots, N)
$$

i.e., if the state of the network never changes as a result of evolution. We usually call the stable state as the attractor of the network.

Let $X$ be a stable state of the network, if $X^{m}$ $(t)=X$ for any integer $t$ (including $t \rightarrow \infty$ ), we say that $X^{m}(0)$ is attracted by $X$, and the state set $\left\{X^{u}(0) \mid X^{u}(t)=X, u=1,2, \ldots, \Lambda\right\}$, which are attracted by $X$, is the attractor basin of $X$.

In associative memory, the weight matrix $W$ is generally designed with Hebbian algorithm. That is, to store $M$ patterns $X^{k}=\{0,1\}^{N}, k=1,2, \ldots$, $M$, in a $N$-neurons network, the synaptic weight matrix is

$$
\begin{gathered}
W_{i j}=\frac{1}{M} \sum_{k=1}^{M}\left(2 X_{i}^{k}-1\right)\left(2 X_{j}^{k}-1\right)-\delta_{i j} \\
i, j=1,2, \ldots, N \\
\delta_{i j}=\left\{\begin{array}{ll}
1 & i=j \\
0 & i \neq j
\end{array}, \quad \text { which ensures } W_{i j}=0 .\right.
\end{gathered}
$$

Here, we convert firstly the mono-polar pattern into bipolar one, then define the synaptic weight matrix of the network as the summation of the out-products of the corresponding bipolar patterns. So, let $V^{k}=2 X^{k}-1$, there is,

$$
W=\frac{1}{M} \sum_{k=1}^{M} V^{k}\left(V^{k}\right)^{t}-I,
$$

$I$ is identify matrix.

It can be easily verified that either $W_{i i}=0$ or 1 has the same properties discussed in this paper. But we prefer the model of DHNN that remains $W_{i i}=0$ for the two reasons: (1) It is proved that DHNN with symmetric zero-diagonal real weight matrix will evolve to a stable state or a limited cycles whose period is 2 for any initial state in synchronous mode [6]. (2) When $W_{i i}=0$, the network is easy to be implemented for the applications.

\section{PERMUTATION SYMMETRY OF DHNN}

The permutation can be expressed in terms of matrix called permutation matrix, which is a 
double-stochastic square matrix of $(0,1)$ and have only one 1 in each row or column. For example, consider $q=(i j)$, which may conveniently be written as:

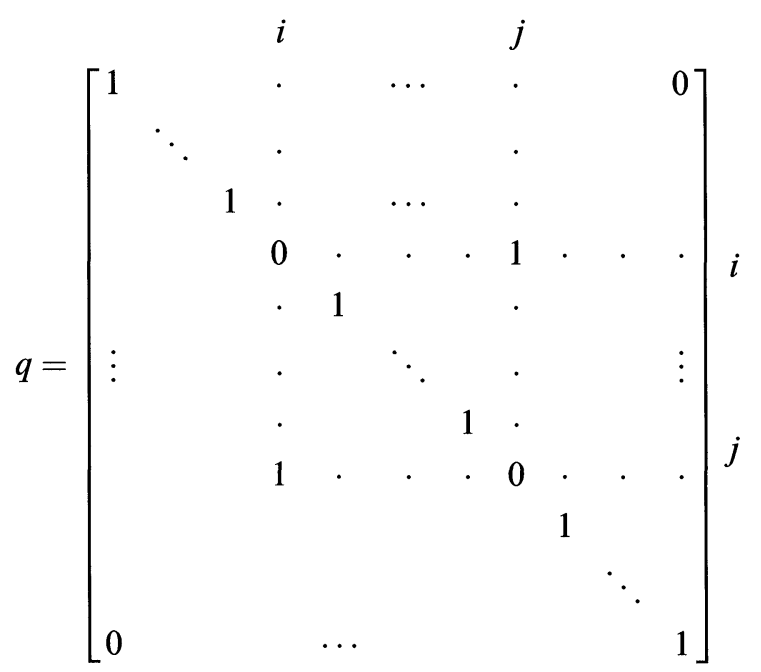

Let $A_{N \times 1}$ be a column vector and $C_{N \times N}$ be a $N \times N$ matrix, then the action of permutation $q=(i j)$ on them would be

$q A_{N \times 1}$, exchange the value of column $i$ with column $j$.

$q C_{N \times N} q^{t}$, exchange the value of column $i$ with column $j$ and row $i$ with row $j$.

Where $q^{t}$ is the transposed matrix of $q$.

An important property of the permutation matrix used in this paper is

$$
q q^{t}=I, \quad \text { i.e., } \quad q^{t}=q^{-1},
$$

where $q^{-1}$ is the inverse matrix of $q$.

When the synaptic weight matrix $W$ of a network $H$ is operated by a random permutation matrix $q$, the relation between $H$ and the new network $\hat{H}$ whose synaptic matrix $\hat{W}=q W q^{t}$ can be described by the following theorem.

Theorem 1 Let $H$ and $\hat{H}$ be two discrete Hopfield neural networks whose synaptic weight matrices are $W$ and $\hat{W}$ respectively such that $\hat{W}=q W q^{t}$. Let the set of attractors of the network $H$ be denoted as $\Psi_{H}=\left\{V^{u}, u=1,2, \ldots, M\right\}$. Let the attractor basin of $H$ be denoted as $\Omega_{H}^{u}=\left\{X^{u v}, v=1, \ldots, \Lambda\right\}$. Then the set of attractors of the network $\hat{H}$ must be $\psi_{\hat{H}}=\left\{\hat{V}^{u}=q V^{u}, u=1,2, \ldots, M\right\}$ and the attractor basin of $\hat{V}^{u}$ must be $\hat{\Omega}_{\hat{H}}^{u}=\left\{\hat{V}^{u v}=q V^{u v}\right.$, $v=1, \ldots, \Lambda\}$.

Proof There are two parts to this theorem.

(1) Let an attractor of the network $H$ denoteed as $V^{u}$, i.e., $V^{u}=\operatorname{step}\left(W V^{u}\right)$. It can be rewritten by $V^{u}=\operatorname{step}\left(W V^{u}\right)=F_{W}\left(V^{u}\right)$. Then,

$$
\begin{aligned}
F_{\hat{W}}\left(\hat{V}^{u}\right) & =F_{\hat{W}}\left(q V^{u}\right)=\operatorname{step}\left(\hat{W} q V^{u}\right) \\
& =\operatorname{step}\left(q W q^{t} q V^{u}\right)
\end{aligned}
$$

$\because q^{t} q=1$ and $\operatorname{step}(q x)=q \operatorname{step}(x)$ where $x$ is any $N \times 1$ matrix.

$$
\begin{aligned}
\therefore \quad F_{\hat{W}}\left(\hat{V}^{u}\right) & =F_{\hat{W}}\left(q V^{u}\right)=q \cdot \operatorname{step}\left(W V^{u}\right) \\
& =q V^{u}=\hat{V}^{u} .
\end{aligned}
$$

So, $\hat{V}^{u}=q V$ must be an attractor of the network $\hat{H}$.

Assume there is an attractor $\hat{V}^{m}$ of the network $\hat{H}$, i.e., $F_{\hat{W}}\left(\hat{V}^{m}\right)=\operatorname{step}\left(\hat{W} \hat{V}^{m}\right)=\hat{V}^{m}$, and $\hat{V}^{m} \notin \Psi_{\hat{H}}=\left\{\hat{V}^{u}=q V^{u}\right\}, \quad$ thus $q^{t} \hat{V}^{m} \notin \Psi_{H}=$ $\left\{V^{u}\right\}$. Otherwise, there is,

$$
\begin{aligned}
F_{W}\left(q^{t} \hat{V}^{m}\right) & =\operatorname{step}\left(W q^{t} \hat{V}^{m}\right)=\operatorname{step}\left(q^{t} \hat{W} q q^{t} \hat{V}^{m}\right) \\
& =q^{t} \operatorname{step}\left(\hat{W} \hat{V}^{m}\right)=q^{t} \hat{V}^{m}
\end{aligned}
$$

i.e., $q^{t} \hat{V}^{m} \in \Psi_{H}=\left\{V^{u}\right\}$. It is opposite.

So, only $\Psi_{\hat{H}}=\left\{\hat{V}^{u}=q V^{u}, u=1,2, \ldots, M\right\}$ is the set of attractors of the network $\hat{H}$.

(2) Let $X(t)$ and $\hat{X}(t)$ respectively denote the trajectories of the dynamical system of $H$ and $\hat{H}$ from the initial state $X(0)$ and $\hat{X}(0)$. Consider $\hat{X}(0)=q X(0)$, thus,

$$
\begin{aligned}
\hat{X}(1) & =F_{\hat{W}}(\hat{X}(0))=F_{\hat{W}}(q X(0)) \\
& =\operatorname{step}(\hat{W} q X(0))=\operatorname{step}\left(q W q^{t} q X(0)\right)
\end{aligned}
$$


$\because q^{t} q=1$ and $\operatorname{step}(q x)=q \operatorname{step}(x)$ where $x$ is any $N \times 1$ matrix.

$$
\begin{aligned}
\therefore \hat{X}(1) & =\operatorname{step}(q W X(0)) \\
& =q \operatorname{step}(W X(0))=q X(1)
\end{aligned}
$$

Similarly, if $\hat{X}(t)=q X(t)$, then $\hat{X}(t+1)=$ $q X(t+1)$. So, if $\hat{X}(0)=q X(0), \quad \hat{X}(t+l)=$ $q X(t+1)$ exists for any integer $t$.

Let $X^{u v}$ denote an initial state converged to the attractor of $V^{u}$ in the network $H$, i.e., $V(0)=X^{u v}$ and $V(t)=F_{W}^{(t)}\left(X^{u v}\right)={ }_{t \rightarrow \infty} V^{u}$.

If $\hat{V}(0)=q V(0)=q X^{u v}$, we have

$$
\begin{aligned}
F_{\hat{W}}^{(t)}\left(q X^{u v}\right) & =\hat{V}(t)=q V(t)=F_{W}^{(t)}\left(q X^{u v}\right) \\
& ={ }_{t \rightarrow \infty} q V^{u}
\end{aligned}
$$

Then, the state in $\hat{\Omega}_{\hat{H}}^{u}=\left\{\hat{V}^{u v}=q V^{u v}, \quad v=\right.$ $1, \ldots, \Lambda\}$ must converge to the attractor of $\hat{V}^{u}$ in the network $\hat{H}$.

Assume there is an initial state $\hat{X}$ in the state space of $\hat{H}$ can converge to $\hat{V}^{u}=q V^{u}$ (i.e., $\hat{V}(t)=F_{\hat{W}}^{(t)}$ $(\hat{X})=_{t \rightarrow \infty} \hat{V}^{u}=q V^{u}$ ) and $\hat{X} \notin \hat{\Omega}_{H}^{u}=\left\{\hat{V}^{u v}=q V^{u v}\right\}$, thus $q^{t} \hat{X} \notin \Omega_{H}^{u}=\left\{X^{u v}\right\}$.

Otherwise, similarly processed as above, let $V(t)$ and $\hat{V}(t)$ respectively denote the trajectories of the dynamical system $H$ and $\hat{H}$ from the initial state $V(0)$ and $\hat{V}(0)$, and consider $V(0)=q^{t} \hat{V}(0)$. Thus,

$$
\begin{aligned}
V(1) & =F_{W}(V(0))=F_{W}\left(q^{t} \hat{V}(0)\right) \\
& =\operatorname{step}\left(W q^{t} \hat{V}(0)\right)=\operatorname{step}\left(q^{t} \hat{W} q q^{t} \hat{V}(0)\right) \\
& =q^{t} \operatorname{step}(\hat{W} \hat{V}(0))=q^{t} \hat{V}(0)
\end{aligned}
$$

Similarly, if $V(t)=q^{t} \hat{V}(t)$ then $V(t+1)=q^{t}$ $\hat{V}(t+1)$.

So, if $V(0)=q^{t} \hat{V}(0), V(t)=q^{t} \hat{V}(t)$ is existence for any integer $t$.

$\because \hat{V}(0)=\hat{X}$ and $\hat{V}(t)=F_{\hat{W}}^{(t)}\left(q^{t} \hat{X}\right)={ }_{t \rightarrow \infty} \hat{V}^{u}=$ $q V^{u}$, and we let $V(0)=q^{t} \hat{V}(0)=q^{t} \hat{X}$.

$$
\begin{aligned}
\therefore F_{W}^{(t)}(V(0)) & =F_{W}^{(t)}\left(q^{t} \hat{X}\right)=V(t)=q^{t} \hat{V}(t) \\
& =q^{t} F_{\hat{W}}^{(t)}(\hat{X})={ }_{t \rightarrow \infty} q^{t} \hat{V}^{u} \\
& =q^{t} q V^{u}=V^{u}
\end{aligned}
$$

i.e., $q^{t} \hat{V}(0) \in \Omega_{H}^{u}=\left\{X^{u v}\right\}$ which is opposite to $q^{t} \hat{X} \notin \Omega_{H}^{u}=\left\{X^{u v}\right\}$. This means only $\hat{\Omega}_{\hat{H}}^{u}=\left\{\hat{V}^{u v}=\right.$ $\left.q V^{u v}, v=1, \ldots, \Lambda\right\}$ can be attracted by $\hat{V}^{u}$ in the network $\hat{H}$.

Thus the theorem is proven.

Theorem 1 describes the variance of the network after performed a random permutation operation. However, there exist some permutations so that the network keeps invariance after permuted by them. Those permutations are the permutation symmetries of the network.

Before studying the permutation symmetry of the network, we introduce firstly some definitions.

Definition 1 The symmetric permutation of the patterns set $\Psi=\left\{X^{u}, u=1,2, \ldots, M\right\}$ is defined as the permutation $q$ which maps patterns set $\Psi$ to itself, $\Psi^{\prime}=\left\{q X^{u}, u=1,2, \ldots, M\right\}=\Psi$. The symmetric permutation of a network with the synaptic weight matrix $W$ is defined as the permutation $g$ such that $g W g^{t}=W$.

Definition 2 A patterns set $\Psi=\left\{X^{u}, u=1\right.$, $2, \ldots, M\}$ is storable [8] if all $M$ sample patterns can be the stable states of some network with the synaptic weight matrix $W$ which is a zero-diagonal real symmetric matrix.

Definition 3 The Hamming weight $w(V)$ of the $N$-dimension binary vector $V=\{1,0\}^{N}$ is defined as the number of component 1 in the vector $w(V)=\sum_{i=1}^{N} V_{i}$.

In Hebbian neural network, the permutation symmetries of the network can be studied by the stored patterns set. We establish the following theorem to show the relationship between them.

THEOREM 2 Let $G$ be the symmetric permutations set of the sample patterns set $\Psi=\left\{X^{u} \mid X^{u}=\right.$ $\left.\left(X_{1}^{u}, X_{2}^{u}, \ldots, X_{N}^{u}\right)^{t}, u=1,2, \ldots, M\right\}$, i.e., $G=\{q / q$ $\Psi=\Psi\}$, where $X^{u}=\{0,1\}^{N}$ and $q$ is a permutation matrix. For $\forall X^{i}, \forall X^{j} \in \Psi$, if there exists at least one permutation $q \in G$ such that $q X^{i}=X^{j}$, we have

(1) All patterns in $\Psi$ have the same Hamming weight.

(2) All of the permutations in $G$ are the symmetric permutations of the network with the synaptic 
weight matrix $W$ which is stored the patterns of $\Psi$ by Hebbian rule.

(3) All patterns in $\Psi$ are stable in the network $(W)$ and have same size of attractor basins if only one of the patterns is stable in the network.

(4) $G$ is a group which is a subgroup of $S_{n}$ consisting of all permutations of the elements from the set $\{1,2, \ldots, n\}$.

\section{Proof}

(1) Let $X^{u}$ and $X^{m}$ belong to patterns set $\Psi$, and their Hamming weight be $K$ and $L$ respectively. Let the permutation relation of them be $q$ such that $q X^{u}=X^{m}, q=\left(\begin{array}{cccc}1 & 2 & \ldots & N \\ i_{1} & i_{2} & \ldots & i_{N}\end{array}\right)$, which replace the component order of $X^{u}$ $(1,2, \ldots, N)$ with $\left(i_{1}, i_{2}, \ldots, i_{N}\right)$, i.e.,

$$
\begin{aligned}
X^{m} & =q X^{u}=q\left(X_{1}^{u}, X_{2}^{u}, \ldots, X_{N}^{u}\right)^{t} \\
& =\left(X_{i_{1}}^{u}, X_{i_{2}}^{u}, \ldots, X_{i_{N}}^{u}\right)^{t} .
\end{aligned}
$$

Assume $K \neq L$, i.e., $w\left(V^{m}\right) \neq w\left(V^{u}\right)$. Otherwise, there is,

$$
w\left(V^{m}\right)=w\left(q V^{u}\right)=\sum_{i_{k}=1}^{N} V_{i_{k}}^{u} .
$$

Because the summation of the components of a vector immaterial to their orders. So,

$$
w\left(V^{m}\right)=\sum_{i_{k}=1}^{N} V_{i_{k}}^{u}=\sum_{i=1}^{N} V_{i}^{u}=w\left(V^{u}\right),
$$

i.e., $K=L$, which is opposite to the assumption.

Therefore, all patterns in $\Psi$ have the same Hamming weight.

(2) To store the patterns of $\Psi$ in a network, the synaptic weight matrix is

$$
W_{i j}=\frac{1}{M} \sum_{u=1}^{M}\left(2 X_{i}^{u}-1\right)\left(2 X_{j}^{u}-1\right)-\delta_{i j}
$$

For convenience, we let $V^{u}=2 X^{u}-1$, then

$$
\begin{aligned}
W & =\frac{1}{M} \sum_{k=1}^{M} V^{k}\left(V^{k}\right)^{t}-I \\
& =\frac{1}{M}\left[V^{1}\left(V^{1}\right)^{t}+V^{2}\left(V^{2}\right)^{t}+\cdots+V^{M}\left(V^{M}\right)^{t}\right]-I
\end{aligned}
$$

For $\forall q \in G$, i.e., $\Psi^{\prime}=\left\{q X^{u}\right\}=\Psi$, we let $\hat{W}$ be the weight matrix that stores the patterns of $\Psi^{\prime}=\left\{q X^{u}\right\}$.

$$
\begin{gathered}
\hat{W}=\frac{1}{M}\left[q V^{1}\left(q V^{1}\right)^{t}+q V^{2}\left(q V^{2}\right)^{t}\right. \\
\left.+\cdots+q V^{m}\left(q V^{m}\right)^{t}\right]-I \\
=\frac{1}{M}\left[q V^{1}\left(V^{1}\right)^{t} q^{t}+q V^{2}\left(V^{2}\right)^{t} q^{t}\right. \\
\left.+\cdots+q V^{m}\left(V^{m}\right)^{t} q^{t}\right]-q I q^{t} \\
=q\left\{\frac { 1 } { M } \left[V^{1}\left(V^{1}\right)^{t}+V^{2}\left(V^{2}\right)^{t}\right.\right. \\
\left.\left.\quad+\cdots+V^{m}\left(V^{m}\right)^{t}\right]-I\right\} q^{t} \\
=q W q^{t}
\end{gathered}
$$

So, $q$ is the symmetric permutation of the weight matrix $W$.

Similarly, all the permutations of $G$ are the symmetric permutation of the weight matrix storing the given patterns set $\Psi$. That is, $G$ is the symmetric permutation of the network.

(3) Let $X^{m} \in \Psi$ be an attractor of the network whose weight matrix $W$ is storing the patterns set $\Psi$ i.e.,

$$
\begin{aligned}
W_{i j} & =\frac{1}{M} \sum_{k=1}^{M}\left(2 X_{i}^{k}-1\right)\left(2 X_{j}^{k}-1\right)-\delta_{i j} \\
X^{m} & =\operatorname{step}\left(W X^{m}\right) .
\end{aligned}
$$

Consider another vector $X^{u} \in M$. Let the permutation relationship between $X^{m}$ and $X^{u}$ be $X^{u}=q X^{m}, q \in G$. We have

$$
\operatorname{step}\left(W X^{u}\right)=\operatorname{step}\left(W q X^{m}\right)
$$

$\because q$ is a symmetric permutation of the network, i.e., $W=q W q^{t}$.

$$
\begin{aligned}
\operatorname{step}\left(W X^{u}\right) & =\operatorname{step}\left(q W q^{t} q X^{m}\right)=\operatorname{step}\left(q W X^{m}\right) \\
& =q \cdot \operatorname{step}\left(W X^{m}\right)=q X^{m}=X^{u}
\end{aligned}
$$

i.e., $X^{u}$ is an attractor of the network.

Similarly, we can prove that all patterns in $\Psi$ are the attractors of the network, i.e., the patterns set $\Psi$ is storable. 
(4) In the permutation set $G$, the product of two permutations $p q$ is defined as the resultant permutation of first permuting with $q$ and then with $p$. Now we prove that the following postulates are satisfied.

Closure i.e., if $q$ and $p$ belong to $G$, then $c=q p$ also belong to $G$.

$\because$ Both $q$ and $p$ are the symmetric permutations of $\Psi$ i.e., $p \Psi=\left\{p X^{u}\right\}=\left\{X^{u}\right\}, q \Psi=\left\{q X^{u}\right\}=\left\{X^{u}\right\}$.

$\therefore c \Psi=q p \Psi=\left\{q p X^{u}\right\}=\left\{q X^{u}\right\}=\left\{X^{u}\right\}$, i.e., $c$ is the symmetric permutation of $\Psi$. so $G$ satisfies the postulate of closure.

Associativity We know from group theory that this postulate of associativity is true for any permutation, i.e., $c(q p)=(c q) p$, for any permutations $c, q, p$. Obviously, $G$ also satisfies this postulate.

Identity Element $e$ The permutation matrix of $e$ is an identity matrix, so $e q=q e=q$ holds for any permutation. So do the permutations in $G$. Therefore, $G$ satisfies this postulate.

Inverse Element We know that $q^{-1}=q^{t}$ is true for any permutation.

For any symmetric permutation $q$, we have,

$$
\begin{gathered}
q^{-1} q \Psi=q^{-1} \Psi, \\
\because q^{-1} q=e \\
\therefore q^{-1} \Psi=e \Psi=\Psi
\end{gathered}
$$

So $q^{-1}$ is also the symmetric permutation of $\Psi$, i.e., $q^{-1} \in G$.

The four postulates are satisfied for $G$. Therefore, $G$ is a group, and accurately, subgroup of the symmetry group $S_{n}$ consisting of all permutations of the elements from the set $\{1,2, \ldots, n\}$.

Thus, the theorem is proved.

In Theorem 2, we give a condition of permutation symmetry that makes sure a set of patterns be storable and have a same basin size of attraction, which can be a selecting rule of the storing patterns set in associative memory, especially for special use of it.

\section{APPLICATION}

In associative memory, the main task is to design a weight matrix to store the given patterns set through a certain learning process, so that the stable states of the network are exactly the given patterns set. If some of the given patterns are not the stable states of the network, we can say that the network loses some memories. If the state $V$ is a stable state of the network but doesn't belong to the given patterns set, we say that $V$ is a spurious stable state of the network. Both losing memories and existence of spurious stable states are the main problems of associative memory. And the former mainly results from the reason that the stored patterns set is not storable, e.g., the similarity of the stored patterns may cause this problem. In this section, we demonstrate how to construct a storable patterns set by the method of group theory.

It is well known that the point group can summerize perfectly the rotation symmetries of the geometric graph in Euclidean space. And any point group is a subgroup of the symmetry group $S_{n}$. So we can use the rotation symmetries of the geometric graph to construct the storable patterns set described in Theorem 2.

For example, there are 24 rotation symmetries in the cube. The 24 rotation symmetries consist of a group called hexahedron group which is isomorphic to $S_{4}$. We can name randomly the vertexes of the cube with $\{1,2, \ldots, 8\}$ (see Fig. 1). Then a symmetric rotation of the cube will correspond to a

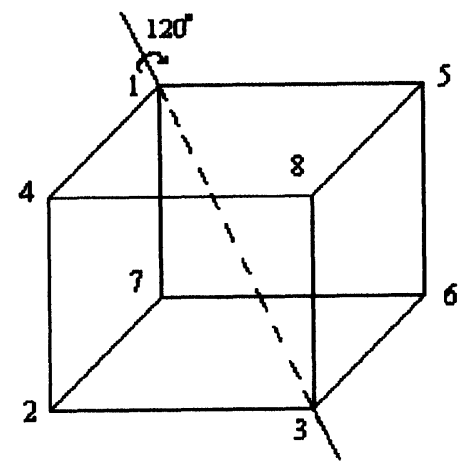

FIGURE 1 The cube. 
permutation of the vertex's index. For example, when we rotate the cube $120^{\circ}$ around the axes 13 , we can see that the cube occupies the same position in the space, but the vertexes' position are changed, which amount to a permutation operation (475) (826).

However, some interesting things would be found. If we put one vector on the cent of each plane of the cube, there are 4 numbers around every vector. Let the component corresponding to the 4 numbers be 1 and the others be 0 , then the 6 vectors will be $\left\{A^{1}=(1,0,0,1,1,0,0,1), A^{2}=(0,0,1,0,1\right.$, $1,0,1), A^{3}=(0,1,1,1,0,0,0,1), A^{4}=(1,0,0,0,1$, $1,1,0), A^{5}=(1,1,0,1,0,0,1,0), A^{6}=(0,1,1,0,0$, $1,1,0)\}$. When connected with some lines, an octahedron with 6 vertexes denoted by the 6 vectors would be seen (see Fig. 2). We can know from group theory that the rotation symmetries of the octahedron are exactly that of the cube. So a symmetric rotation of the octahedron is related to a symmetric permutation of the 6 vectors. In addition, we can see from Figure 2 that any vertex can be replaced by another through rotating the octahedron. That is, in the set of the 6 vectors, any vector can be obtained from another by a symmetric permutation. So the set of vectors is storable according to Theorem 2.

We can examine it by constructing a network with the 6 vectors. Computed by Eq. (3). the synaptic weight matrix is

$W=\left[\begin{array}{cccccccc}0 & -1 / 3 & -1 & 1 / 3 & 1 / 3 & -1 / 3 & 1 / 3 & -1 / 3 \\ -1 / 3 & 0 & 1 / 3 & 1 / 3 & -1 & -1 / 3 & 1 / 3 & -1 / 3 \\ -1 & 1 / 3 & 0 & -1 / 3 & -1 / 3 & 1 / 3 & -1 / 3 & 1 / 3 \\ 1 / 3 & 1 / 3 & -1 / 3 & 0 & -1 / 3 & -1 & -1 / 3 & 1 / 3 \\ 1 / 3 & -1 & -1 / 3 & -1 / 3 & 0 & 1 / 3 & -1 / 3 & 1 / 3 \\ -1 / 3 & -1 / 3 & 1 / 3 & -1 & 1 / 3 & 0 & 1 / 3 & -1 / 3 \\ 1 / 3 & 1 / 3 & -1 / 3 & -1 / 3 & -1 / 3 & 1 / 3 & 0 & -1 \\ -1 / 3 & -1 / 3 & 1 / 3 & 1 / 3 & 1 / 3 & -1 / 3 & -1 & 0\end{array}\right]$

For the writing convenience, we list all the attractors and their domains of attraction by their transposed forms in Table I.

We can see from Table I that the patterns set consisting of the 6 vectors is storable and all the 6 vectors have a same basin size of attraction.

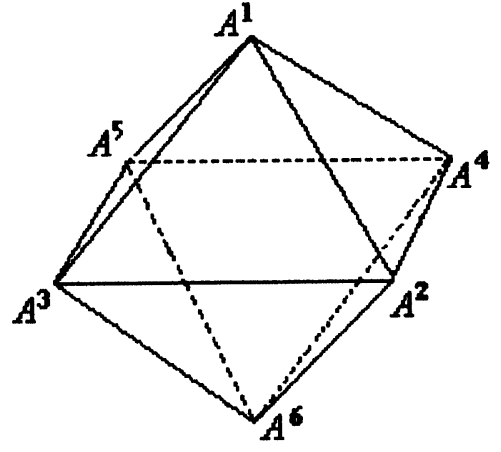

FIGURE 2 The octahedron.

TABLE I The classification table of the network with the synaptic matrix Eq. (7). In each column, vector beyond the line is attractor, vectors below the line form a basin of attraction

\begin{tabular}{llllll}
\hline$A^{1}$ & $A^{2}$ & $A^{3}$ & $A^{4}$ & $A^{5}$ & $A^{6}$ \\
\hline
\end{tabular}

$100110010010110101110001 \quad 100011101101001001100110$ $\begin{array}{llllll}00100011 & 0000011100010011 & 00011001 & 00110001 & 00001011\end{array}$ 001001100000110100010110000111000011010000001110 010001100001010101010010010110000100001101001010 $\begin{array}{llllll}01001100 & 00100101 & 10010010 & 1000001101001001 & 10000110\end{array}$ 010101000010100111000010100010010101000110001010 011000100010110011001000100100010110000110001100 $01100100 \quad 0010110111010000100110000111000010001110$ 011001100010111111010010100110010111000110001111 $\begin{array}{lllllll}01100111 & 00111101 & 11010011 & 1001101101110011 & 10010100\end{array}$ $0110111001101000110101101001110101110101 \quad 10011110$ 011101100110110111011010101000010111100110100100 $10100010101010001110000010111001 \quad 1011000010101110$ 111001101010110111110010110110011111000111001110

In this example, we show that the storable patterns set can be constructed by an octahedral group with the aid of geometric graph in Euclidean space. However, it can easily be generalized to the other group.

\section{DISCUSSION AND CONCLUSION}

Discrete Hopfield neural network was proposed mainly as an associative memory model. Hopfield demonstrated by computer simulation that the network with $N$ neurons can store about $0.138 \mathrm{~N}$ patterns in the form of the stable states, that is, not any patterns set is storable. One may thinks that 
the main reasons for the small capacity is the dynamic system of the network itself, and some researchers try to improve the capacity by modifying the dynamic system of the network, such as the dynamical behavior [9], the connecting dynamics [10,11], the output function [12], and so on. But results are not satisfactory well. All those show that a storable patterns set is necessary for associative memory, especially for special use of it.

In addition, it should also be noticed that all the prototypes built to this date, in optical or very large-scale integrated technology, are still of a fairly small size. So, it is necessary to derive exact properties for the network as opposed to asymptotic or to noncompletely rigorous ones, such as those which are based on mean-field theory in statistical mechanics which may be valid only in the limit of large systems.

In this paper, we study the discrete Hopfield neural network by the method of permutation, and associate the permutation symmetries of the network with its storing patterns set. By this way, we find some interesting properties in DHNN, which can be applied to associative memory in selecting a storable patterns set. It can be easily verified that the well-known Hadamard patterns set [13] omitted the trivial pattern $(0,0, \ldots, 0)$, whose permutation symmetries are proved to be isomorphic to the group $G L\left(n, F_{2}\right)$ by Folk [14], satisfies the condition proposed in Theorem 2 . So, the Hadamard network, in which the Hadamard patterns set has been stored by Hebbian learning rule, can be regarded as a special case in this paper.

\section{References}

[1] Eiliot, J. P. and Dawber, P. G. (1979). Symmetry in Physics (London: Macmillan).

[2] Lautrup, B. (1989). The Hopfield Model (Singapore: World Scientific).

[3] Baldi, P. (1987). Phys. Rev. Lett., 59, 1976.

[4] Baldi, P. (1988). SIAM J. Disc. Math., 1(1).

[5] Hopfield, J. J. (1982). Proc. Natl. Acad. Sci., USA[C], 79, $2554-2558$.

[6] Ma Jinwen (1997). Neural Networks, 10, 1109-1116.

[7] Jin-quan Chen (1989). Group representation theory for physicists. (Singapore: World Scientific).

[8] Ma Jinwen (1999). Neural Computing and Applications, 8, 25-32.

[9] Morita, M. (1993). Neural Networks, 6, 115-126.

[10] Venkatesh, S. S. and Psaltis, D. (1989). IEEE Trans on Information Theory, 35, 558-568.

[11] Ott, P. and Lueder, E. (1994). In: Proc. The 6th Microcomputer Workshop, Neural Networks Theory and Applications, Sedmihorky, Tschechische Republick, pp. 218-224.

[12] Marcus, C. M. et al. (1991). Physica D, 51, 234-247.

[13] Krisenment, O. (1990). Z Physik B, 80, 415.

[14] Folk, R., Kartashov, A., Lisonek, P. and Paule, P. (1993). J. Phys. A: Math., 26, 3159-3164. 


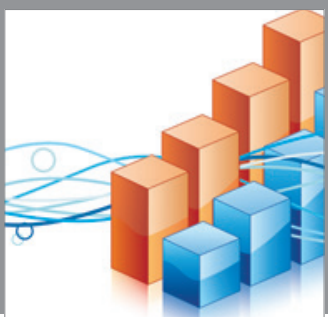

Advances in

Operations Research

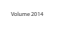

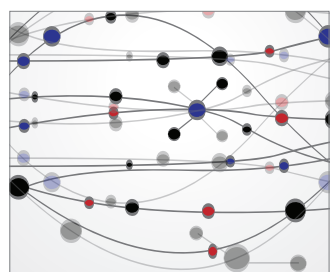

\section{The Scientific} World Journal
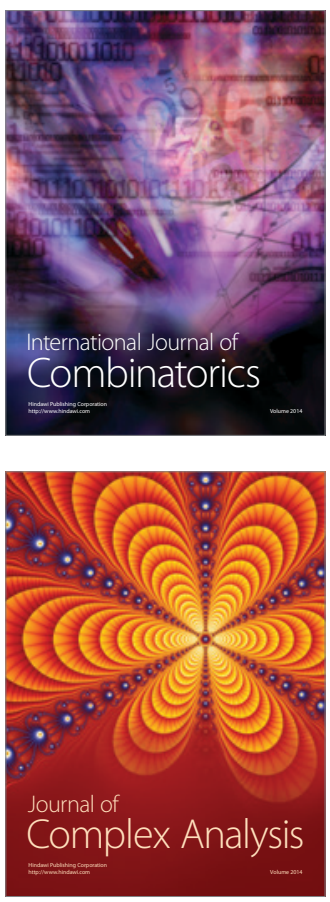

International Journal of

Mathematics and

Mathematical

Sciences
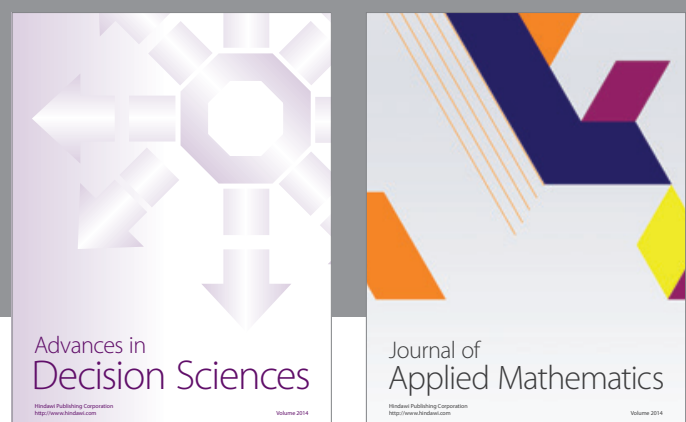

Journal of

Applied Mathematics
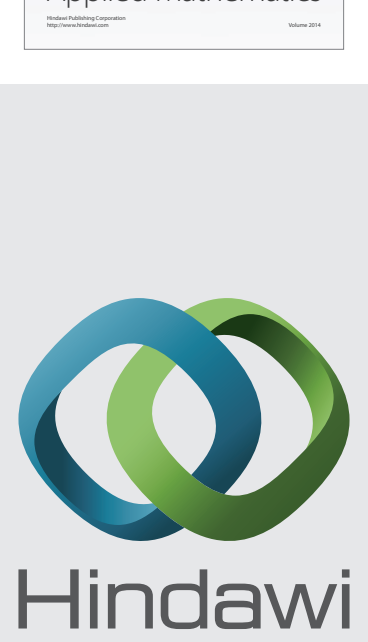

Submit your manuscripts at http://www.hindawi.com
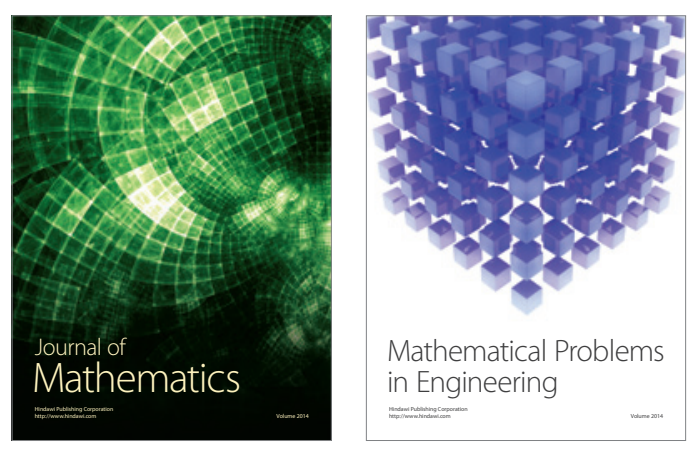

Mathematical Problems in Engineering
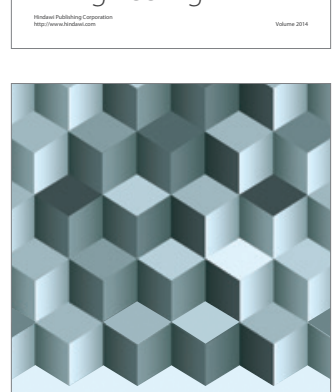

Journal of

Function Spaces
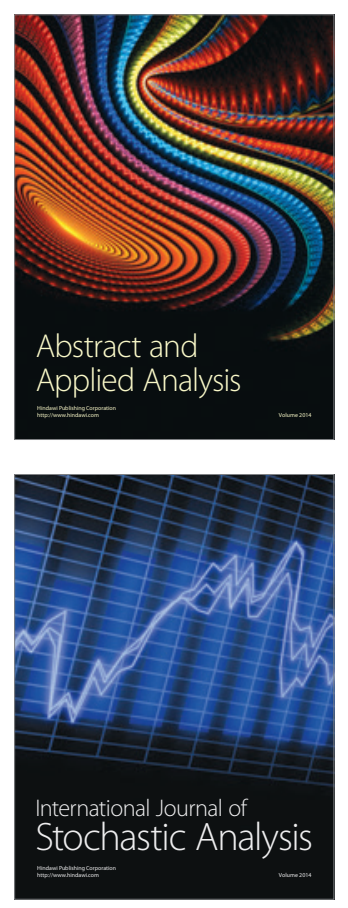

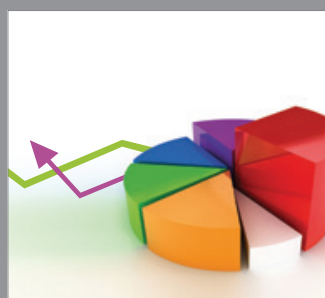

ournal of

Probability and Statistics

Promensencen
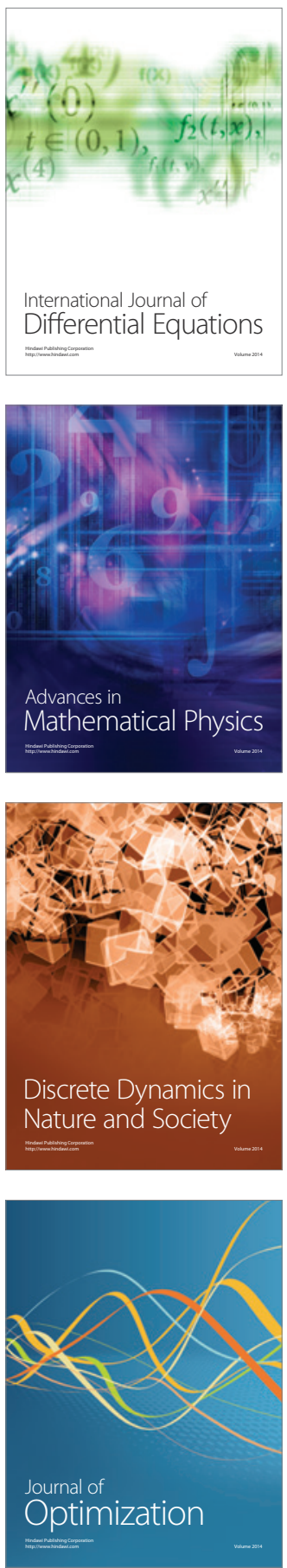